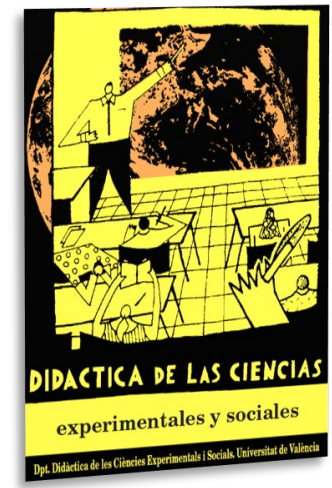

\title{
Enfoques para la enseñanza de la Biología: una mirada para los contenidos
}

\author{
Approaches to teaching Biology: a look at the \\ contents
}

\author{
Gonzalo Miguel Angel Bermudez \\ Universidad Nacional de Córdoba - CONICET (Argentina), gbermudez@unc.edu.ar \\ ORCID iD: https://orcid.org/0000-0001-9734-0965
}

\author{
Maricel Occelli \\ Universidad Nacional de Córdoba - CONICET (Argentina), maricel.occelli@unc.edu.ar \\ ORCID iD: http://orcid.org/0000-0002-4516-0644
}

\begin{abstract}
RESUMEN: En el marco de un diseño didáctico multirreferencial y contextualizado a necesidades específicas, tomar decisiones sobre los contenidos exige poner en juego conocimientos específicos de la didáctica de las ciencias. Tras relevar el uso del término enfoque en la bibliografía de didáctica de las ciencias experimentales y formación del profesorado, reconocemos como enfoques establecidos a CTSA, la interdisciplinariedad y la historia de las ciencias. Sin embargo, al conceptualizar nuestra postura acerca del concepto de enfoque, asociado a las decisiones que tomamos las y los docentes sobre los contenidos, realizamos un análisis epistemológico y didáctico de la Biología y de propuestas de enseñanza de esta disciplina. Con base en ello proponemos y describimos ocho enfoques más para la enseñanza de contenidos biológicos, que diversifican los tres enfoques anteriores: morfo-funcional, taxonómico-clasificatorio, evolutivo, sistémico y ecológico, educación ambiental y educación para el desarrollo sostenible, diversidad cultural, educación en salud y educación sexual integral.
\end{abstract}

Palabras ClaVe: CTSA, educación ambiental, diversidad cultural, educación en salud, educación sexual.

ABSTRACT: Within the framework of a multi-referential didactic design and contextualized to specific needs, making decisions about the contents requires putting into play specific knowledge of science teaching. After reviewing the use of the term approach in the literature of didactics of experimental sciences and teacher training, we recognized as established approaches STSE, interdisciplinarity and the history of science. However, when conceptualizing our position on the concept of approach, associated with the decisions that teachers make about contents, we carried out an epistemological and didactic analysis of Biology and of teaching proposals of this discipline. Based on that, we propose and describe eight approaches more to teach biological contents, which diversify the three previous ones: morpho-functional, taxonomic-classificatory, evolutionary, systemic and ecological, environmental education and education for sustainable development, cultural diversity, health education, and comprehensive sexual education.

KEYWORDS: STSE, environmental education, cultural diversity, health education, sex education.

Fecha de recepción: marzo de 2020

Fecha de aceptación: julio de 2020

Agradecemos a la Universidad Nacional de Córdoba, Secretaría de Ciencia y Tecnología y a la Agencia Nacional de Promoción Científica y Tecnológica de Argentina, FONCYT (proyecto PICT-2015-1903) por la financiación, y a la Mg. Priscila Ariadna Biber por sus aportes sobre Educación en Salud y Sexual Integral. 


\section{INTRODUCCIÓN}

En toda planificación didáctica definir qué se va enseñar requiere de un proceso reflexivo analítico y de toma de decisiones. En este artículo deseamos aportar, desde algunas reflexiones teóricas vinculadas a la didáctica de las ciencias, diferentes enfoques que pueden guiar la selección de contenidos y de actividades para la enseñanza de la biología.

Un primer aspecto que deseamos explicitar es nuestra posición acerca de la tarea de enseñar, la cual la entendemos como una actividad crítica y humana, una práctica social impregnada de opciones de carácter ético, en la cual los valores presiden su intencionalidad (Litwin, 2008). En particular, pensar en la selección de contenidos hace referencia a este proceso del planeamiento educativo que nos lleva a decidir acerca de ¿qué enseñar? Esta elección se realiza para un contexto en particular y desde una mirada docente específica en la cual se ponen en juego diversas cuestiones que aluden a posicionamientos, saberes, experiencias y valores. De manera que un diseño didáctico es una síntesis creativa, producto de un conjunto de decisiones de carácter multirreferencial (Alliaud, 2017; Souto, 2017). Proponemos retomar como analogía la imagen de una urdimbre para pensar los diseños didácticos, ya que nos permite imaginar el modo en que estas múltiples referencias se entrecruzan en un tejido. Por ejemplo, ¿qué Biología enseñar? ¿qué modelo de ciencias biológicas quiero enseñar? ¿cómo hacer para responder a los principios esenciales y actualizados de la Biología y a la vez a los diversos intereses y necesidades sociales, culturales y ambientales? Así, un hilo que tensa a este tejido o diseño nos lleva a plantearnos interrogantes acerca de lo que entendemos como buena enseñanza (Fenstermacher, 1989).

En el proceso de diseño didáctico, los saberes sobre una determinada práctica social, como pueden ser las prácticas científicas de Biología, son seleccionados y adaptados a nuevas funcionalidades en el proceso de transposición (Bravo, 2005). Sin embargo, y retomando la idea de la urdimbre, no todo tejido da lugar a un proceso en relación con las problemáticas del ámbito sociocultural del estudiantado (Beltrán Véliz, Aburto y Troncoso, 2018). Esta perspectiva propone que la selección de contenidos no tiene una respuesta única, sino que por el contrario habrá múltiples posibles respuestas en función del mapa de saberes, concepciones y valores de cada docente. En este sentido, Izquierdo Aymerich (2005) plantea que "los contenidos pasan a ser una variable respecto a la cual se han de tomar decisiones; es decir, un 'problema' para la didáctica de las ciencias" (p.112). Es por ello que, a fin de ofrecer caminos de aproximación para la toma de decisiones didácticas, en este artículo aportamos una redefinición teórica para el concepto de enfoque en función de su utilización en el contexto de la didáctica de las ciencias específicamente, y desarrollamos un conjunto de enfoques para la enseñanza de contenidos de Biología, los que pueden tomarse y modificarse para otras ciencias experimentales y sociales.

\section{EL USO DEL TÉRMINO ENFOQUE EN LA BIBLIOGRAFÍA DE LA FORMACIÓN DEL PROFESORADO Y LA EDUCACIÓN EN CIENCIAS: HACIA UNA REDEFINICIÓN}

El término enfoque es actualmente usado en la literatura de la enseñanza de las ciencias y la formación del profesorado, aunque sin ofrecer una conceptualización precisa del mismo. En general, enfoque alude a una forma de mirada o una perspectiva particular, de modo cercano a su significado desde los aportes de la pedagogía, y en particular, encontramos que su uso coincide con los aportes de Fenstermacher y Soltis (2007), quienes refieren a los enfoques de la enseñanza para argumentar cómo diversas formas de entender la educación se corresponden con modos disímiles de desarrollar prácticas de enseñanza. Por su parte, Pontes Pedrajas, Poyato López y Oliva Martínez (2016) clasifican las creencias sobre el aprendizaje de profesores en formación según tres enfoques: uno tradicional o transmisivo, uno intermedio y uno constructivista.

En este artículo proponemos circunscribir a los enfoques en términos didácticos, y específicamente desde la didáctica de las ciencias. En función de la variedad de perspectivas con la 
que se utiliza este término, y tras un proceso de microanálisis, hemos podido identificar tres grupos definidos y establecidos de propuestas teóricas y desarrollos didácticos anclados en los contenidos: (a) el enfoque histórico-epistemológico o historia de las ciencias, (b) el enfoque de CienciaTecnología-Sociedad-Ambiente (CTSA), y (c) el enfoque interdisciplinar.

Con respecto al primero, la Historia de la Ciencia (HDC) es uno de los enfoques más idóneos para abordar aspectos de la Naturaleza de la Ciencia (NDC), como, por ejemplo, las finalidades, objetivos y contextualización de las investigaciones, el papel de las comunidades científicas en la construcción y validación de las ideas, etc. (Acevedo-Díaz y García-Carmona, 2016). Un modo eficaz de implementar el enfoque HDC es a partir de la lectura de narraciones de casos y controversias suscitadas con hechos que han promovido la construcción de saberes científicos pasados. Entre los ejemplos anclados en este enfoque histórico-epistemológico, encontramos los trabajos Acevedo-Díaz y García-Carmona (2016), sobre el caso de Rosalind Franklin para la elucidación de la estructura molecular del ADN, y de Aragón-Méndez, García-Carmona y Acevedo-Díaz (2016), quienes proponen actividades sobre distintos aspectos de las investigaciones de Semmelweis sobre la fiebre puerperal.

La perspectiva denominada Ciencia, Tecnología, Sociedad (CTS) es otra corriente establecida en la educación científica a la que suele hacerse referencia como un enfoque, y ha venido renovando el currículo gracias a la búsqueda de relaciones entre los desarrollos científicos y tecnológicos y los procesos sociales. Este enfoque pretende superar el carácter neutral atribuido habitualmente a la ciencia y, desde una perspectiva histórica y compleja, destacar su papel dinamizador en el desarrollo de la sociedad (García-Carmona y Criado, 2008). En su desarrollo, CTS ha tomado diversas nominalizaciones $\mathrm{y}$, en la actualidad, parece haber un consenso en la forma CTSA, que incluye explícitamente al ambiente $(A)$ con el fin de destacar la necesidad de una conciencia ambiental (Pérez y Lozano, 2013). Las Cuestiones Sociocientíficas (CSC) toman gran relevancia ya que se constituyen en casos que exponen en contextos reales las relaciones CTSA. Las CSC plantean discusiones en las cuales interactúan, en conversación, un colectivo compuesto por diferentes actores y fuerzas sociales. Estas situaciones a menudo se encuentran en las fronteras del conocimiento científico e implican tomar una postura ante discusiones sobre costos, beneficios y riesgos, lo ético y los valores. Existen múltiples ejemplos de contenidos biológicos que se vinculan a CSC, tales como los riesgos vinculados con la producción y el uso de los organismos genéticamente modificados, la utilización de tecnologías reproductivas, el uso de células madres, la pérdida de la biodiversidad, etc. (Bermudez y De Longhi, 2015; Massarini y Schnek, 2015; Occelli, Garcia Romano y Valeiras, 2018). Con base en lo anterior, identificamos numerosas contribuciones a la enseñanza de la Biología que se adscriben a un enfoque CTS. Por ejemplo, Grilli Silva (2015), organiza los contenidos y actividades de un curso universitario de zoología para que los estudiantes adquieran una mirada de cuestiones cotidianas con un soporte desde la construcción humana de la ciencia. A su vez, de Toledo y Camero (2015) realizan una propuesta para la enseñanza de los sistemas respiratorio, circulatorio y digestivo a través de actividades que reconocen el rol de la tecnología para llevar a cabo diagnósticos y terapias de enfermedades.

En tercer lugar, la forma de abordar el currículum de forma interdisciplinaria representa otro núcleo de investigaciones y desarrollos que buscan integraciones entre disciplinas, más o menos afines, al tratar de resolver problemas complejos que exceden la mirada desde un campo en particular (Vázquez-Alonso y Manassero-Mas, 2017). En el aula, la interdisciplinariedad ayuda a que los estudiantes alcancen habilidades de pensamiento crítico y de resolución de situaciones problemáticas (Czerniak y Johnson, 2014). En el campo curricular ha ganado fuerza, en los últimos años, el constructo STEM (science-technology-engineering-mathematics / ciencia-tecnología-ingenieríamatemática), lo que se visualiza en reformas curriculares (Couso, 2017; Czerniak y Johnson, 2014). Como ejemplo de un diseño didáctico desde la perspectiva interdisciplinar mencionamos que la propuesta sobre alimentación de Carretero Gómez (2006), a partir del análisis de las comidas mencionadas en la obra El Quijote, relaciona las ciencias sociales, la educación física, la matemática 
y la lengua. Si bien identificamos que algunos aportes en educación ambiental y patrimonial suelen ser enmarcados en la interdisciplinariedad (Morales Hernández, Caurín Alonso, Sendra Mocholí y Parra Monserrat, 2014), consideramos que la EA constituye un enfoque diferente y, por tanto, será considerado con mayor detalle más adelante en este artículo.

Si bien estos enfoques tienen una larga tradición pueden no ser los únicos para abordar contenidos. ¿Hay otras formas de abordaje del curriculum que, si bien son reconocidas en las prácticas, no son identificadas aún como enfoques?

\section{NUESTRA APROXIMACIÓN A LA DEFINICIÓN DE ENFOQUE: UNA PERSPECTIVA DIDÁCTICA PARA LA TOMA DE DECISIONES CURRICULARES}

La didáctica de las ciencias desarrolla criterios para la selección y organización de conocimientos escolares que puedan funcionar en un sistema didáctico, de acuerdo con las expectativas sociales y educativas actuales (Izquierdo Aymerich, 2005). Este sistema se planifica en unidades didácticas, para las que las y los profesores realizamos tareas como: (a) decidir la temática que se trabajará con el alumnado; (b) seleccionar y organizar los contenidos que se enseñarán; (c) seleccionar y secuenciar las actividades; y (d) diseñar la evaluación (Rivero, Fernández y Rodríguez, 2013). Si nos concentramos en lo referido a los contenidos, desde los años ' 80 se han dado debates en el ámbito de la didáctica de las ciencias que han derivado en constructos y orientaciones prácticas respecto a los criterios de selección, la organización y secuenciación, la aproximación disciplinar, etc. (Caamaño, 2013). Algo similar ha venido ocurriendo con la reflexión didáctica acerca de las actividades seleccionadas para el estudiantado, clasificando su momento (inicio, desarrollo, etc.), jerarquía (tareas, estrategias), semejanza con las prácticas científicas de referencia (indagación, modelización), contexto en el que tienen lugar (salida de campo, etc.), etc. (Amórtegui, Mayoral y Gavidia, 2017; Gomez Galindo, 2014).

Sin embargo, con el fin de integrar muchas de estas decisiones, podemos considerar la orientación que toma el diseño didáctico a través de énfasis dado a ciertos contenidos y actividades para lograr una finalidad determinada (Izquierdo Aymerich, 2005). Así, De Longhi, Bernardello, Crocco y Gallino (2003) expresan que cada docente toma decisiones sobre qué contenidos enseñar y con qué enfoque lo hará. En dicho trabajo, los autores refieren al enfoque desde su uso cotidiano, el que puede definirse como la acción y efecto de "dirigir la atención o el interés hacia un asunto o problema desde unos supuestos previos, para tratar de resolverlo acertadamente" (Real Academia Española $^{1}$ ). A su vez, De Longhi et al. (2003) especifican que el/la profesor(a) puede optar por una aproximación disciplinar o interdisciplinar, o "si el enfoque será histórico, evolutivo, sistémico o taxonómico, entre otras decisiones" (p. 25).

En el presente trabajo retomamos dichas expresiones para conceptualizar didácticamente al enfoque como la perspectiva con la que se mirarán los contenidos y se seleccionarán las actividades en función de objetivos concretos, considerando que, en su conjunto, los aprendizajes que emergen de estas decisiones pueden agruparse en función de núcleos epistemológicos ${ }^{2}$. Estos núcleos concentran prácticas y saberes culturales diversos, principalmente, el de la actividad (científica) de referencia, y pueden servir al profesorado para orientar su enseñanza. Por ello, la identificación del enfoque permitiría al docente explorar y caracterizar las lógicas con la que se dan diferentes prácticas y determinar situaciones de enseñanza que las vinculen (Fuchs-Gallezot y Coquidé, 2010).

Desde la perspectiva didáctica que proponemos usaremos como analogía para el enfoque el hecho de centrar una imagen en el visor de una cámara o los filtros que se utilizan para la publicación de fotografías en las redes sociales, dando nitidez a ciertos objetos y tornando borrosos a otros (ponderando así diferentes aspectos de la trama epistemológica).

\footnotetext{
${ }^{1} \mathrm{https}: / /$ dle.rae.es/?id=FIJ8jj4

${ }^{2}$ El sentido de epistemología es el que refiere no sólo a aspectos gnoseológicos, sino también sintácticos, semánticos, praxeológicos y axiológicos (Adúriz-Bravo y Erduran, 2003).
} 
Dependiendo de la temática y la consideración de criterios que se establezcan serán más pertinentes unos enfoques que otros. A su vez, el contexto también permitirá dilucidar qué enfoque puede resultar más adecuado, teniendo en cuenta el nivel educativo, tipo de institución, grupo de estudiantes, etc.

\section{Caracterización de otros ENFOQues didácticos PARA espacios CURRICULARES DE BIOLOGÍA}

\subsection{Enfoque morfo-funcional}

Centra su perspectiva en la composición y funciones biológicas de los seres vivos, enfatizando la visión sincrónica de la Biología (Bermudez, 2015). Por ejemplo, si los contenidos tratasen de la anatomía y fisiología del cuerpo humano, sus partes y su organización celular, en tejidos, órganos y sistemas, el enfoque morfofuncional prioriza la conceptualización de los niveles de organización en una jerarquía determinada, identificando partes y sus funciones. Una gran parte de los modelos científicos de las Ciencias Biológicas que se transponen en contenidos destinados a la enseñanza en la escuela secundaria versan sobre la dimensión organizativa de la vida, con cierto énfasis en el reconocimiento de nombres de estructuras. Sin embargo, Adúriz-Bravo y Erduran (2003) advierten que el centrismo en la nomenclatura y taxonomía puede implicar un modelo de la disciplina biológica de carácter ateórico, con reminiscencias del positivismo lógico del Círculo de Viena. Como ejemplo de contenidos y actividades que podrían ubicarse bajo este enfoque, Bahamonde y Galindo (2016) abordan la enseñanza de la digestión humana a través de una modelización, con la que conceptualizan las relaciones estructura-función en tramos del tubo digestivo y órganos anexos. En otra propuesta, Baranzelli et al. (2014) relatan una experiencia en educación primaria en la que estudian los diferentes aparatos bucales de los insectos y su funcionamiento en relación con sus hábitos alimenticios.

\subsection{Enfoque taxonómico-clasificatorio}

Este abordaje se centra en el pensamiento clasificatorio, la importancia de la delimitación de unidades de expresión y de sus criterios de comparación. Al igual que el enfoque morfo-funcional, el taxonómico-clasificatorio comprende principalmente aquello delimitado a la Biología funcional o sincrónica (Bermudez, 2015), puesto que la idea de categoría no implica una noción de evolución o desarrollo individual (ver enfoque evolutivo más adelante), sino que agrupa a entidades características similares. A pesar de que bajo el paradigma evolutivo las relaciones filogenéticas (ancestralidad común) son consideradas por la comunidad científica como el aspecto más útil para delimitar las especies y clasificar a los organismos (Rosa y Tricarico, 2016), el parentesco no constituye el único criterio de clasificación. En relación con ello, lo taxonómico-clasificatorio permite desarrollar contenidos relativos al componente más conspicuo de la biodiversidad (PérezHarguindeguy, Enrico y Díaz, 2015).

Es importante que en el trabajo desde este enfoque no sean reforzadas posiciones esencialistas, ya que investigaciones didácticas han remarcado que este tipo de pensamiento representa un obstáculo epistemológico para el aprendizaje de la evolución (González Galli, Meinardi y Pérez, 2018). Con base en lo anterior, reconocemos que la unidad didáctica de Mengascini (2005) posee un enfoque taxonómico-clasificatorio y propone la reconstrucción de una clasificación de tejidos vegetales según su función y sus caracteres citológicos. También identificamos bajo este enfoque la contribución de Vilches et al. (2012), quienes abordan el estudio de las adaptaciones a la forma de vida de los moluscos bivalvos. 


\subsection{Enfoque evolutivo}

Pone el foco en la transformación de los componentes y relaciones de los sistemas biológicos en el tiempo, reparando tanto en las causas de los cambios como en sus consecuencias. Los contenidos que se relacionan más estrechamente con este enfoque son los de transformación y evolución (visión diacrónica), los que, a su vez, han servido al establecimiento de la Biología como ciencia (Castro Moreno y Valbuena Ussa, 2018). El reconocimiento de la diversidad como producto del proceso evolutivo provocó un cambio en las clasificaciones, ya que la pertenencia a un taxón y las relaciones jerárquicas entre los mismos se basan exclusivamente en el parentesco filogenético (Arana, Correa y Oggero, 2014; Pérez-Harguindeguy et al., 2015). Un ejemplo de actividades que podemos ubicar bajo este enfoque son las que proponen Pérez, Gómez-Galindo y Gónzalez-Galli (2018), quienes presentan una secuencia didáctica basada en la modelización y en la metacognición con el fin de superar obstáculos para el aprendizaje de la evolución biológica. El trabajo de Rosa y Tricarico (2016) también está anclado en un enfoque evolutivo, puesto que emplea árboles evolutivos para estudiar la transformación de las plantas en el tiempo.

\subsection{Enfoque sistémico y enfoque ecológico}

Fomenta el abordaje de las interrelaciones entre las partes de un sistema y, sobre todo, las propiedades emergentes de las diferentes configuraciones, ya que, aunque ciertas características estén ausentes en los elementos básicos, éstas se manifiestan a partir de las interacciones entre dichos componentes (Mayr, 2006). A causa de su complejidad, los sistemas biológicos poseen capacidades tales como "la reproducción, el metabolismo, la replicación, la regulación, la adaptación, el crecimiento y la organización jerárquica" (Mayr, 2006, p.46). Otro aspecto añadido sobre los sistemas complejos es la necesidad de pensar en múltiples niveles interdependientes y la causalidad no lineal. Así, los objetos de estudio de la Ecología y las Ciencias de la Tierra son especialmente fértiles para ser abordados con un enfoque sistémico (Morin, 2002).

Con base en lo anterior, Cañal (2008) propone un esquema conceptual sistémico y relacional de las relaciones y funciones entre órganos y sistemas a nivel del organismo humano. Desde este enfoque sistémico, Galagovsky y Greco (2009) proponen un diseño didáctico para abordar las propiedades emergentes de los sistemas biológicos a través de una secuencia de actividades centrada en un modelo didáctico analógico.

Por otro lado, dado el avance de la Ecología como ciencia, creemos que el enfoque sistémico podría adquirir tintes particulares cuando se toman decisiones sobre contenidos y actividades relacionadas con los ecosistemas. En este sentido, interpretamos que la propuesta didáctica de Rodellar y Bravo-Torija (2016) está organizada con un enfoque ecológico, ya que implementa tareas que giran en torno a la elaboración de un plan de regeneración de una zona de bosque dañada tras un incendio. En otro ejemplo, Gual Oliva (2013) propone una unidad didáctica en la que los estudiantes diseñan acciones para mejorar la calidad ambiental de su ciudad.

\subsection{Enfoque de educación ambiental (EA) y educación para el desarrollo sostenible (EDS)}

Desde que surgiera el uso del término Educación Ambiental (EA) en 1948, y su rápida expansión en la década de los 60's, el campo de la EA ha cambiado tanto como las problemáticas ambientales que dieron origen a su surgimiento (Dillon, 2014). Desde sus primeros pasos, la EA buscó generar una ciudadanía con conocimientos y valores sobre entornos biofísicos y socioculturales, promoviendo el respeto por el medio ambiente, y que sea comprometida con el desarrollo de habilidades y actitudes necesarias para el trabajo de búsqueda de soluciones a problemas ambientales (Vázquez Cano, 2012). En la actualidad es posible reconocer que la EA ha venido transitando un camino desde una orientación de tinte positivista hacia paradigmas socio-críticos, los 
que contemplan cómo los factores políticos y contextuales afectan la vida de las personas (Dillon, 2015). En este marco, el presente de la EA está caracterizado por un amplio espectro de posiciones teóricas que van desde finalidades propedéuticas y formas tradicionales de actividades a prácticas de reciclaje escolar y activismo ambiental. De hecho, desde los 80's han surgido tensiones entre el estatus de la EA y el campo de la educación en ciencias (Hart, 2015).

Algo similar a lo ocurrido con EA viene sucediendo desde la declaración de Tbilisi de la Organización de las Naciones Unidas para la Educación, la Ciencia y la Cultura (UNESCO) acerca de las nociones de sostenibilidad. En este sentido, el concepto de desarrollo sostenible (DS) fue descrito por primera vez en el Informe de la Comisión Bruntland de 1987 como "el desarrollo que satisface las necesidades actuales sin comprometer la capacidad de las futuras generaciones de satisfacer sus propias necesidades" (Comisión Mundial sobre el Ambiente y el Desarrollo, 1987, citado en Hart, 2015). El DS ha planteado desde sus orígenes una agenda que incluye la reducción de la pobreza, el cambio de los patrones de consumo, el crecimiento de la población mundial y la protección de la salud humana, por lo que se presentan importantes desafíos para los sistemas sociales y económicos actuales (UNESCO, 2012, citado en Hart, 2015).

Si bien la EDS conservó credibilidad oficial en documentos y declaraciones, proporcionando un impulso a las nociones públicas de DS, según Dillon (2014), la comunidad de la EA consideró a la EDS más bien como una apropiación discursiva de la resistencia en la política medioambiental y del desarrollo. Estas tensiones han animado la discusión académica acerca de las relaciones entre EA y EDS, por ejemplo, entre quienes consideran su solapamiento, que EDS forma parte de la EA, o que EDS es una etapa en la evolución de EA. Sobre esta última posición, Dillon (2014) explica que quienes promulgan la superioridad de la EDS refieren a que es más amplia que la EA, ya que incluye cuestiones más básicas que las metas de esta última, tales como el mejoramiento de la calidad y el acceso a la educación (Agenda 21) (Dillon, 2014). Más allá de estas consideraciones, Hart (2015) advierte que EA y EDS están actuando en conjunto y por separado como prácticas socioecológicas y materiales que, en la mayoría de los sistemas educativos, se han incorporado al currículo de ciencias como "pequeños detalles añadidos" (Hart, 2015, p.388).

Reconocemos que el proyecto didáctico de Vázquez Cano (2012) en una escuela secundaria está comprometida con el logro de objetivos coherentes con una combinación de EA y EDS. Por otro lado, la propuesta educativa de aulas abiertas de Polop (2018), que promueve un modelo de conservación y educación sobre el valor del patrimonio natural y cultural del bosque, se organiza en actividades que persiguen la consecución de logros coherentes con un enfoque de EA.

\subsection{Enfoque de diversidad cultural}

Frecuentemente denominada perspectiva o clave, las referencias a la diversidad cultural en la enseñanza de las ciencias involucran aspectos políticos, epistémicos, ontológicos e ideológicos interrelacionados, presentes en los debates acerca del cientificismo, del conocimiento científico y el reconocimiento de la otredad en el proceso de enseñanza (Andrade, Rivera, Suárez y Ríos, 2009; Pérez-Mesa, 2013, 2019). Reconocer a la cultura como un sistema de significados implica tener en cuenta las formas de enunciar y significar la realidad, donde lo cultural se constituye en un recurso heurístico para hablar de la diferencia en la búsqueda de la negociación de los significados y el cruce de fronteras (Pérez Mesa, 2019).

La educación en general, y la enseñanza de las ciencias en particular, ha considerado al conocimiento científico como el sistema de referencia para evaluar otras perspectivas, desconociéndolas y negándolas. Sin embargo, tal y como sostienen Andrade y Mojica (2013), la cultura científica y el conocimiento cotidiano o tradicional son epistemes diferentes, están basadas en algún tipo de racionalidad y en formas de conocer y de producir conocimiento útil para cada cultura. Por ello, trabajar desde un enfoque de diversidad cultural genera compromisos y aperturas, principalmente en lo que se refiere a la actitud orientadora de una acción abierta a la diferencia y lo 
heterogéneo; y la discusión sobre una perspectiva que asuma la condición de lo diverso en relación con lo cognitivo, las concepciones y cosmovisiones del sujeto (Andrade et al., 2009).

Creemos importante distinguir que la tarea didáctica de indagar y explicitar concepciones sobre una temática a enseñar, para recuperarlas a modo de ideas previas o conocimiento cotidiano, no califica como trabajo desde la diversidad cultural, principalmente por el estatus que, en este caso, tienen en el aula. El abordaje desde un enfoque de diversidad cultural implica que el reconocimiento de la diferencia y la otredad es tanto objetivo como contenido de enseñanza. Es decir, evitar el etnocentrismo epistemológico implica que la construcción del conocimiento escolar se apropie de criterios de inclusión y de pluralidad, y establezca puentes entre el conocimiento tradicional y el conocimiento científico occidental (Pérez Mesa, 2019).

Reconocemos bajo el enfoque de diversidad cultural al trabajo de González-García y ContrerasFernández (2013), quienes, tras estudiar la taxonomía botánica presente en el mapudungun, proponen la inclusión de plantas medicinales empleadas por la etnia mapuche en una carrera universitaria. A su vez, Bernal, Andrade y Melo (2018) dan cuenta del desarrollo de un proyecto didáctico en educación primaria con un enfoque cultural, en el que, a través de actividades en contextos como la huerta y plaza del mercado, analizan el caso de la papa como puente epistemológico entre conocimientos.

\subsection{Enfoque educación en salud (EeS)}

En la bibliografía se utiliza una pluralidad de articulaciones para relacionar Educación y Salud, cada una respondiendo a sentidos diferentes. Aquí acordamos con Martins (2019) en la utilización de la denominación Educación en Salud (EeS) para superar perspectivas verticalistas o de salud individual a las que se asocian otras formas de vincular estos conceptos. La autora caracteriza la EeS como un proceso político-pedagógico cuyo objetivo es aumentar la autonomía de los individuos en la toma de decisiones acerca de cuestiones individuales y colectivas que involucran a la salud. Este enfoque prioriza el desarrollo de conocimientos, habilidades, destrezas y actitudes para que las personas transformen y participen en el mundo en el que viven, siendo protagonistas y promotoras de comportamientos saludables.

En el ámbito de la EeS se pueden identificar perspectivas que refieren a conceptos de salud muy diferentes (Gavidia y Talavera, 2012). En este artículo, desde una mirada multicausal y multireferencial, adherimos a una concepción de salud procesual producto de una relación dialéctica entre seres humanos y el ambiente, y que puede comprenderse como una adaptación diferencial al ambiente (Revel Chion, Meinardi y Adúriz-Bravo, 2013). Considerando esta perspectiva de salud holística, social, colectiva y en íntima relación con el ambiente, proponemos al enfoque EeS desde la promoción de la salud desde el cual se promueve el análisis crítico y reflexivo, y se facilita la concientización y empoderamiento de la salud a través de una toma de decisiones personales y colectivas saludables (Buss, 2006).

Enseñar Biología desde la promoción de la salud requiere realizar una lectura crítica y profunda de los contextos socioculturales e institucionales de modo que se construyan propuestas contextualizadas de EeS, fruto de un posicionamiento reflexivo. En la práctica, ello implica analizar, recrear y generar propuestas para escenarios educativos concretos, contemplando las subjetividades del estudiantado y los desafíos sociales y culturales del momento (Pastorino, Astudillo y Rivarosa, 2016).

Se registran numerosas propuestas didácticas que trabajan contenidos de Biología desde la EeS. Rivadulla-López, García-Barros y Martínez-Losada (2016) presentan una aproximación histórica al concepto de nutrición humana, y sugieren criterios para organizar y secuenciar los contenidos a partir de problemas estructurantes. Por otro lado, Uskola, Burgoa y Maguregi (2018) proponen la modelización de una situación en contexto, la que expone a la vacunación como temática 
sociocientífica, brindando oportunidades para desarrollar un juicio crítico y la toma de decisiones vinculadas al sistema inmunológico.

\subsection{Enfoque educación sexual integral (ESI)}

Esta perspectiva plantea una formación en los derechos ciudadanos para el desarrollo pleno de la sexualidad, el respeto y la posibilidad de elegir. La educación sexual integral (ESI) se propone como un pilar para la construcción de infancias y juventudes libres, diversas, con igualdad de derechos y oportunidades en pleno ejercicio de su libertad (Ayuso, 2019). Asumiendo que toda educación es sexual, y tomando como referencia una perspectiva de género y de derechos humanos, entendemos a la ESI como aquella educación que articula aspectos biológicos, psicológicos, sociales, afectivos y éticos. Comprender a la ESI desde la perspectiva crítica de género implica analizar las relaciones de poder/saber vinculadas con la construcción de los cuerpos sexuados. Por su parte, la perspectiva de derechos humanos es la que orienta desde un parámetro de justicia para una práctica política (Morgade, Fainsod, González del Cerro y Busca, 2016). Este enfoque pone en cuestión la producción y reproducción de las relaciones sexogenéricas, con lo cual todas las dimensiones de las prácticas educativas se ven interpeladas, inclusive los contenidos, como es el foco de este artículo. En este sentido, y focalizando nuestra mirada en la enseñanza de la Biología, surgen cuestionamientos vinculados a qué Biología enseñar. Por la necesidad de sintetizar, resaltaremos aquí solo dos cuestiones que este enfoque nos invita a considerar.

Una primera cuestión se encuentra vinculada a la no neutralidad del conocimiento científico, es decir, las relaciones de poder y los estereotipos de género que tienen lugar en su construcción. Así, se puede analizar la producción de conocimiento científico sobre métodos de anticoncepción, los cuales se han focalizado en la medicalización sobre los cuerpos considerados femeninos y la ausencia de métodos farmacéuticos de anticoncepción para varones cisheterosexuales (Grotz, 2019). La no neutralidad también se evidencia al trabajar la menstruación superando ideas negativas a las que usualmente se la vincula (asco, suciedad y vergüenza) o su asociación directa con la reproducción. Una manera de abordar a la menstruación en su complejidad es incorporando aspectos psicoemocionales y socioculturales que permitan aprender a leer nuestros propios cuerpos y generar autoconocimiento (Kohen y Meinardi, 2016). Sin embargo, el enfoque de la ESI no solo permite trabajar contenidos vinculados a la fisiología humana. En Morgade et al. (2016) se analiza una propuesta para la enseñanza de la evolución humana que presenta el contenido biológico de modo tal que da lugar a discutir formas de desigualdades que atraviesa la construcción social del conocimiento, como sucede cuando esta producción es sesgada por una cultura androcéntrica o machista, pero también racista y eurocentrista.

Un segundo aspecto a considerar en el marco de este enfoque es la necesidad de romper con un pensamiento binario, evitando por ejemplo dos únicas tipologías femenino o masculino para abordar los caracteres sexuales, las hormonas, la dotación cromosómica sexual, etc. (Grotz, 2019). El pensamiento binario invisibiliza las intersexualidades e impone identificaciones de género según dotación biológica, desconociendo que la identificación con lo femenino y lo masculino responde a construcciones sociohistóricas que exceden ampliamente a lo biológico (Granero y García, 2019). Es por ello que desde un enfoque de ESI se propone abordar estos contenidos biológicos desde una perspectiva que busque educar en la diversidad afectiva sexual.

\section{REFLEXIONES FINALES}

Sin la pretensión de abarcar todas las posibilidades de enfoques posibles, en este artículo hemos aportado reflexiones teóricas para delimitar una forma de comprender a los enfoques y desarrollamos ocho opciones diferentes para orientar propuestas de enseñanza de la Biología, además de otras tres, más consolidadas en la educación en ciencias. Tomar la decisión de seleccionar uno de estos enfoques 
como perspectiva a partir de la cual diseñar una propuesta didáctica implica reflexionar en profundidad qué Biología queremos enseñar. Es decir, desde qué lente o filtro proponemos abordar el estudio de una temática, lo cual requiere reflexionar y poner en juego una red de conocimientos, posiciones y valores que son desde las cuales entendemos de modo personal a la tarea de educar. Esta perspectiva reflexiva exige que cada docente construya criterios para poder realizar una toma de decisiones autónoma y para ello se necesita una actitud de indagación y crítica permanente que evite la alienación y rutinización de la práctica y promueva la construcción activa de saberes que refuerce el desarrollo profesional docente (Pastorino et al., 2016).

Los enfoques desarrollados en este artículo intentan ilustrar cómo los contenidos a seleccionar y secuenciar pueden tomar dimensiones divergentes al abordarse desde cada uno de ellos. Consideramos que decidir cuál es el enfoque más pertinente para un diseño didáctico requiere un ejercicio recursivo y dialógico entre las diferentes dimensiones y fundamentos de toda práctica de enseñanza; es decir, que exige hacer visible para sí mismo/a desde qué urdimbre creamos nuestros tejidos. Así, será necesario no solo pensar en los fundamentos de la propia disciplina sino, principalmente, en las oportunidades que cada uno de estos enfoques ofrece al contexto sociocultural específico para el cual estará dirigida la propuesta de enseñanza. En este sentido y dependiendo de cuán profunda sea nuestra reflexión, para seleccionar un enfoque se requiere interpelarse acerca aquella pregunta esencial que compartiera Fenstermacher (1989) acerca de las buenas enseñanzas y que, en definitiva, nos invitan a pensar en cuál es la visión de educación que sostienen nuestras prácticas.

\section{Referencias}

Acevedo-Díaz, J.A. y García-Carmona, A. (2016). Rosalind Franklin y la estructura molecular del ADN: un caso de historia de la ciencia para aprender sobre la naturaleza de la ciencia. Revista Científica, 25, 162-175. DOI: 10.14483/udistrital.jour.RC.2016.25.a2

Adúriz-Bravo, A. y Erduran, S. (2003). La epistemología específica de la biología como disciplina emergente y su posible contribución a la didáctica de la biología. Revista de Educación en Biología, 6(1), 9-14.

Alliaud, A. (2017). Los artesanos de la enseñanza. Acerca de la formación de maestros con oficio. Buenos Aires: Paidós.

Amórtegui, E.F.C., Mayoral, O.G.B. y Gavidia, V.C. (2017). Aportaciones de las Prácticas de Campo en la formación del profesorado de Biología. Didáctica de las Ciencias Experimentales y Sociales, 32(1), 153-170. DOI: 10.7203/DCES.32.9940

Andrade, A.M. y Mojica, L. (2013). Enseñanza como puente entre conocimientos científicos escolares y conocimientos ecológicos tradicionales. Magis. Revista Internacional de Investigación en Educación, 6(12), 37-53. DOI: 10.11144/Javeriana.m6-12.ecpe

Andrade, A.M., Rivera, C.A.M., Suárez, C.J.M. y Ríos, L.M. (2009). Diversidad cultural e implicaciones en la enseñanza de las ciencias: reflexiones y avances. Revista Colombiana de Educación, (56), 106-130. DOI: 10.17227/01203916.7582

Arana, M.D., Correa, A.L. y Oggero, A.J. (2014). El reino plantae: ¿qué es una planta y como se clasifican?. Revista de Educación en Biología, 17(1), 9-24. Recuperado a partir de https://revistas.psi.unc.edu.ar/index.php/revistaadbia/article/viewFile/22413/22031

Aragón-Méndez, M.M., García-Carmona, A. y Acevedo-Díaz, J.A. (2016). Aprendizaje de estudiantes de secundaria sobre la naturaleza de la ciencia mediante el caso histórico de Semmelweis y la fiebre puerperal. Revista Cientifica, 27, 302-317. DOI: 10.14483/udistrital.jour.RC.2016.27.a1

Ayuso, B.A. (2019). Educación Sexual Integral. Construyendo derechos. Revista de Educación en Biología, 21(2), 4-7. Recuperado a partir de https://revistas.unc.edu.ar/index.php/revistaadbia/article/view/24521 
Bahamonde, N. y Galindo, A.A.G. (2016). Caracterización de modelos de digestión humana a partir de sus representaciones y análisis de su evolución en un grupo de docentes y auxiliares académicos. Enseñanza de las Ciencias, 34(1), 129-147. DOI: 10.5565/rev/ensciencias. 1748

Baranzelli, M.C., Córdoba, S., Cocucci, A., Glinos, E., Paiaro, V., Sazatornil, F., Sérsic, A. y Wiemer, A.P. (2014). Dime cómo comes y te diré quién eres: una experiencia didáctica para conocer los aparatos bucales de los insectos. Revista de Educación en Biología, 17(2), 76-85. Recuperado a partir de https://revistas.unc.edu.ar/index.php/revistaadbia/article/view/22438

Beltrán Véliz, J.C., Aburto, B.N. y Troncoso, S.P. (2018). Prácticas que obstaculizan los procesos de transposición didáctica en escuelas asentadas en contextos vulnerables: Desafíos para una transposición didáctica contextualizada. Revista Educación, 42(2), 335-355. DOI: 10.15517/REVEDU.V42I2.27571

Bermudez, G.M.A. (2015). Los orígenes de la Biología como ciencia. El impacto de las teorías de evolución y las problemáticas asociadas a su enseñanza y aprendizaje. Revista Eureka sobre Enseñanza y Divulgación de las Ciencias, 12(1), 66-90. DOI: 10.25267/Rev_Eureka_ensen_divulg_cienc.2015.v12.i1.06

Bermudez, G.M.A. y De Longhi, A.L. (2015). Retos para la enseñanza de la biodiversidad hoy. Aportes para la formación docente. Córdoba: Universidad Nacional de Córdoba.

Bernal, M.C., Andrade, A.M. y Melo, N.B. (2018). Puente contextual, diálogo de conocimientos tradicionales y científicos escolares: El caso de la papa en el grado cuarto de primaria. Tecné Episteme y Didaxis: TED, Número extraordinario. Octavo Congreso Internacional de formación de Profesores de Ciencias. 10 a 12 de Octubre de 2018, Bogotá. pp. 1-9. Recuperado a partir de https://revistas.pedagogica.edu.co/index.php/TED/article/download/9086/6823

Bravo, R.R. (2005). Aproximación al concepto de transposición didáctica. Revista Folios, (21), 3345. Recuperado a partir de https://www.redalyc.org/pdf/3459/345955978004.pdf

Buss, P.M. (2006). Una introducción al concepto de promoción de la salud. En Czeresnia, D. y Freitas, C. M. Promoción de la salud: conceptos, reflexiones, tendencias (pp. 19-46). Buenos Aires: Lugar Editorial.

Caamaño, A. (2013). Hacer unidades didácticas: una tarea fundamental en la planificación de las clases deficiencias. Alambique Didáctica de las Ciencias Experimentales, 74, 5-11. Recuperando a partir de https://dialnet.unirioja.es/servlet/articulo?codigo $=4198149$

Cañal, P. (2008). El cuerpo humano: una perspectiva sistémica. Alambique. Didáctica de las Ciencias Experimentales, 58, 8-22.

Carretero Gómez, M.B. (2006). El Quijote: nutrición y salud. Revista Eureka sobre Enseñanza y Divulgación de las Ciencias, 3(1), pp. 134-157. DOI: 10.25267/Rev_Eureka_ensen_divulg_cienc.2006.v3.i1.10

Castro Moreno, J.A. y Valbuena Ussa, É.O. (2018). Algunas relaciones entre la autonomía de la Biología y la emergencia de su Didáctica: consideraciones sobre la complejidad de enseñar una ciencia compleja. Ciência \& Educação (Bauru), 24(2), 267-282. DOI: 10.1590/1516731320180020002 .

Couso, D. (2017). Per a què estem en STEM? Un intent de definir l'alfabetització STEM per a tothom i amb valors. Revista Ciències, 34, 22-30. DOI: 10.5565/rev/ciencies.403

Czerniak, C.M. y Johnson, C.C. (2014). Interdisciplinary science teaching. In N.G. Lederman y S.K. Abell (Eds.), Handbook of Research in Science Education. Volume II (pp. 395-411). Nueva York: Routledge.

De Longhi, A.L., Bernardello, G., Crocco, L. y Gallino, M. (2003). Genética y evolución. Volumen II. Buenos Aires: Ministerio de Educación de la Nación.

De Toledo, M.O. y Camero, R.E. (2015). Desarrollo de cinco recursos con enfoque CTS para la Enseñanza de Sistema Respiratorio, Circulatorio y Digestivo. Revista de Investigación, 39(85), 63-91. Recuperado a partir de https://www.redalyc.org/pdf/3761/376143541005.pdf 
Dillon, J. (2014). Environmental Education. In N.G. Lederman y S.K. Abell (Eds.), Handbook of Research in Science Education (pp. 497-514). Volume II. New York, NY: Routledge.

Fenstermacher, G.D. (1989). Tres aspectos de la filosofía de la investigación sobre la enseñanza. En: M.C. Wittrock (Ed.), La investigación de la enseñanza: Enfoques, teorías y métodos (pp. 150176). México: Paidós.

Fenstermacher, G.D. y Soltis, J. (2007). Enfoques de la enseñanza. Buenos Aires: Amorrortu.

Fuchs-Gallezot, M. y Coquidé, M. (2010). Génétique, génomique et post-génomique dans les programmes de SVT, une discipline scientifique scolaire. RDST. Recherches en Didactique des Sciences et des Technologies, 2, 17-52. Recuperado a partir de http://rdst.revues.org/272

Galagovsky, L.R. y Greco, M. (2009). Uso de analogías para el "aprendizaje sustentable": El caso de la enseñanza de los niveles de organización en sistemas biológicos y sus propiedades emergentes. Revista Electrónica de Investigación en Educación en Ciencias, 4(1), 10- 33. Recuperado a partir de https://dialnet.unirioja.es/descarga/articulo/2882625.pdf

García-Carmona, A. y Criado, A. M. (2008). Enfoque CTS en la enseñanza de la Energía Nuclear: análisis de su tratamiento. Enseñanza de las Ciencias, 26(1), 107-124. Recuperado a partir de https://www.raco.cat/index.php/Ensenanza/article/view/89261/297675

Gavidia, V. y Talavera, M. (2012). La construcción del concepto de salud. Didáctica de las Ciencias Experimentales y Sociales, 26, 161-175. Recuperado a partir de https://ojs.uv.es/index.php/dces/article/view/1935/1449

Gómez Galindo, A. A. (2014). Progresión del aprendizaje basado en modelos: la enseñanza y el aprendizaje del sistema nervioso. Biografía - Escritos sobre la Biología y su Enseñanza, 7(13), 101-107. DOI: 10.17227/20271034.vol.7num.13bio-grafia101.107

González-García, F. y Contreras-Fernández, D.E. (2013). Diversidad vegetal: de los mapuches a la enseñanza formal chilena. Magis, Revista Internacional de Investigación en Educación, 6(12), 153-167. DOI: 10.11144/Javeriana.m6-12.dvme

González Galli, L.M.G., Meinardi, E.N. y Pérez, G.M. (2018). Una tipología de casos para enseñar el modelo de evolución por selección natural. Didáctica de las Ciencias Experimentales y Sociales, (34), 77-90. DOI: 10.7203/DCES.34.12056

Granero, A. y García, T. (2019). Intersexualidades: desconocimiento teórico-práctico en la educación formal. Profesorado. Revista de Currículum y Formación de Profesorado, 23(1), 61-82. DOI:10.30827/profesorado.v23i1.9144

Grilli Silva, J.J. (2015). Seven Pounds y Biología. Zoología y trasplante de órganos en el epílogo del film, una experiencia en formación de docente. Didáctica de las Ciencias Experimentales y Sociales, (29), 233-246. DOI: 10.7203/DCES.29.3911

Grotz, E. (2019). Ciencias Biológicas y Naturales. En: M. Mirabal (Ed.), Educación Sexual Integral Epistemología, pedagogía y política en los debates curriculares. Cuadernos del IICE No 3 (pp. 49-63). Buenos Aires: Facultad de Filosofía y Letras, UBA.

Gual Oliva, M. (2013). El pensamiento sistémico como vía para avanzar hacia la comprensión de los fenómenos complejos: el caso de los fenómenos ambientales urbanos. Enseñanza de las Ciencias (Número Extra), 1674-1679. Recuperado a partir de https://www.raco.cat/index.php/Ensenanza/article/view/307418

Hart, P. (2015). Environmental education and science education. In R. Gunstone (Ed.), Encyclopedia of Science Education (pp. 384-390). Dordrecht: Springer.

Izquierdo Aymerich, M. (2005). Hacia una teoría de los contenidos escolares. Enseñanza de las Ciencias, 23(1), 111-122. Recuperado a partir de https://www.raco.cat/index.php/Ensenanza/article/view/22008/332750

Kohen, M. y Meinardi, E. (2016). Problematizando las enseñanzas sobre la menstruación en la escuela: lo disimulado, lo negativo, lo silenciado. Biografía - Escritos sobre la Biología y su Enseñanza, 9(16), 179-183. DOI: 10.17227/20271034.vol.9num.16bio-grafia179.183

Litwin, E. (2008). El oficio de enseñar. Condiciones y contextos. Buenos Aires: Paidós. 
Martins, I. (2019). Educação em Ciências e Educação em Saúde: breves apontamentos sobre histórias, práticas e possibilidades de articulação. Ciência \& Educação, 25(2), 269-275. DOI: 10.1590/1516-731320190020001

Massarini, A., y Schenk, A. (Coords.) (2015). Ciencia entre todxs. Tecnociencia en contexto social. Una propuesta de enseñanza. Buenos Aires: Paidós.

Mayr, E. (2006). Por qué es única la biología. Consideraciones sobre la autonomía de una disciplina cientifica. Buenos Aires: Katz.

Mengascini, A. (2005). La enseñanza y el aprendizaje de los tejidos vegetales en el ámbito universitario. Revista Electrónica de Enseñanza de las Ciencias, 4(2), 2-13. Recuperado a partir de http://reec.uvigo.es/volumenes/volumen4/ART4_Vol4_N2.pdf

Mirabal, M. (2019). Educación Sexual Integral Epistemología, pedagogía y política en los debates curriculares. Cuadernos del IICE N ${ }^{\mathrm{o}}$ 3, Buenos Aires: Facultad de Filosofía y Letras, UBA. Recuperado a partir http://repositorio.filo.uba.ar/bitstream/handle/filodigital/11196/Cuaderno\%20IICE\%203.pdf?s equence $=1 \&$ isAllowed $=\mathrm{y}$

Morales Hernández, A.J., Caurín Alonso, C., Sendra Mocholí, C. y Parra Monserrat, D. (2014). Aprendiendo a plantear problemas en el medio. Análisis de una experiencia con estudiantes del Máster de Investigación en Didácticas específicas. Didáctica de las Ciencias Experimentales y Sociales, 28, 65-81. DOI: 10.7203/DCES.28.3848

Morgade, G., Fainsod, P., González del Cerro, C. y Busca, M. (2016). Educación sexual con perspectiva de género: reflexiones acerca de su enseñanza en biología y educación para la salud. Biografias: Escritos sobre la Biología y su Enseñanza, 9(16), 149-167. DOI: 10.17227/20271034.vol.9num.16bio-grafia149.167

Morin, E. (2002). La cabeza bien puesta. Reformar la reforma. Reformar el pensamiento. $1^{\circ}$ ed. Buenos Aires: Nueva Visión.

Occelli, M., Garcia Romano, L. y Valeiras, N. (2018). La enseñanza de la biotecnología y sus controversias socio-científicas en la escuela secundaria: un estudio con profesores de biología la ciudad de Córdoba (Argentina). Tecné, Episteme y Didaxis: TED, 43, 31-46. DOI: 10.17227/ted.num43-8650

Pastorino, I.C., Astudillo, C.S. y Rivarosa, A.S. (2016). Aportes para una didáctica de la Educación para la Salud en la formación inicial de profesores de Biología: diálogos divergentes, concepciones y prácticas. Revista de Educación en Biología, 19(1), 73-82. Recuperado a partir de https://revistas.unc.edu.ar/index.php/revistaadbia/article/view/22531

Pérez, G., Gómez-Galindo, A., y Gónzalez-Galli, L. (2018). Enseñanza de la evolución: fundamentos para el diseño de una propuesta didáctica basada en la modelización y la metacognición sobre los obstáculos epistemológicos. Revista Eureka Sobre Enseñanza Y Divulgación de las Ciencias, 15(2), 2102. DOI: 10.25267/Rev_Eureka_ensen_divulg_cienc.2018.v15.i2.2102

Pérez, L.F.M. y Lozano, D.L.P. (2013). La emergencia de las cuestiones sociocientíficas en el enfoque CTSA. Góndola, Enseñanza y Aprendizaje de las Ciencias, 8(1), 23-35. DOI: $10.14483 / 23464712.5021$

Pérez-Harguindeguy, N., Enrico, L. y Díaz, S. (2015). ¿Qué es la Diversidad Biológica? (y por qué nos importa, cómo se genera y cómo se mide). En G.M.A. Bermudez y De Longhi, A.L. (Coords.), Retos para la enseñanza de la biodiversidad hoy. Aportes para la formación docente (pp. 25-55). Córdoba: Universidad Nacional de Córdoba.

Pérez-Mesa, M. R. (2013). Concepciones de biodiversidad: una mirada desde la diversidad cultural. Magis. Revista Internacional de Investigación en Educación, 6(12), 133-151. DOI: 10.11144/Javeriana.m6-12.cbmd

Pérez-Mesa, M. R. (2019). Concepciones de biodiversidad y prácticas de cuidado de la vida desde una perspectiva cultural. Tecné, Episteme y Didaxis: TED, (45), 17-34. DOI: 10.17227/ted.num45-9830 
Polop, F. J. (2018). El desafío de aprender en aulas abiertas: una propuesta de aprendizaje vivencial y contextual. Revista de Educación en Biología, 21(1), 62-73. Recuperado a partir de https://revistas.unc.edu.ar/index.php/revistaadbia/article/view/22546

Pontes Pedrajas, A., Poyato López, F.J. y Oliva Martínez, J.M. (2016). Creencias sobre el aprendizaje de las ciencias de los estudiantes del máster de profesorado de enseñanza secundaria. Didáctica de las Ciencias Experimentales y Sociales, 30, 137-163. DOI: 10.7203/DCES.31.7881

Revel Chion, A., Meinardi, E. y Adúriz-Bravo, A. (2013). Elementos para un análisis histórico epistemológico del concepto de salud con implicaciones para la enseñanza de la Biología. Filosofia e História da Biologia, 8(1), 1-19. Recuperado a partir de http://www.abfhib.org/FHB/FHB-08-1/FHB-8-1-01-Andrea-Revel-Chion_ElsaMeinardi_Agustin-Aduriz-Bravo.pdf

Rivadulla-López, J.C., García-Barros, S. y Martínez-Losada, C. (2016). Historia de la Ciencia e ideas de los alumnos como referentes para seleccionar contenidos sobre nutrición. Revista Eureka sobre Enseñanza y Divulgación de las Ciencias, 13 (1), 53-66. DOI: 0.25267/Rev_Eureka_ensen_divulg_cienc.2016.v13.i1.05

Rivero, A., Fernández, J. y Rodríguez, F. (2013). ¿Para qué sirven las setas? Diseño de una unidad didáctica en biología para aprender investigando. Alambique, 73, 38-48. Recuperado a partir de https://idus.us.es/handle/11441/41052

Rodellar, G.F. y Bravo Torija, B. (2016). Entre todos salvaremos el monasterio de piedra: una actividad para promover el aprendizaje de ecología y el uso de pruebas en secundaria. Didáctica de las Ciencias Experimentales y Sociales, 30, 117-135. DOI: 10.7203/DCES.30.5152

Rosa, S.M. y Tricarico, H. (2016). Uso de árboles evolutivos para contextualizar científicamente la enseñanza de la biodiversidad vegetal. Revista Eureka sobre Enseñanza y Divulgación de las Ciencias, 13(2), 384-394. DOI: 10.25267/Rev Eureka ensen divulg cienc.2016.v13.i2.11

Souto. M. (2017). Pliegues de la formación. Sentidos y herramientas para la formación docente. Rosario: Ediciones Homo Sapiens.

Uskola, A., Burgoa, B. y Maguregi, G. (2018). Influencia de la ayuda del profesorado en la construcción del modelo de sistema inmunológico y su aplicación en las tomas de decisión. Revista Eureka sobre Enseñanza y Divulgación de las Ciencias, 15(3), 3604. DOI: 10.25267/Rev_Eureka_ensen_divulg_cienc.2018.v15.i3.3604

Vázquez Cano, E. (2012). El tratamiento interdisciplinar de lo eco-sostenible en la enseñanza secundaria: un estudio de casos. Profesorado. Revista de Curriculum y Formación de Profesorado, 16(2), 165-192. Recuperado a partir de http://www.ugr.es/ recfpro/rev162ART10.pdf

Vázquez-Alonso, Á. y Manassero-Mas, M. A. (2017). Interdisciplinariedad y conceptos nómadas en didáctica de la ciencia: consecuencias para la investigación. Revista Eureka sobre Enseñanza y Divulgación de las Ciencias, 14(1), 24-37. DOI: 0.25267/Rev_Eureka_ensen_divulg_cienc.2017.v14.i1.03

Vilches, A., Legarralde, T., Maroñas, M. y Darrigran, G. (2012). Aportes para el trabajo en el aula. Un enfoque para determinar la forma de vida de los moluscos bivalvos a partir de la morfología de sus valvas. Revista de Educación en Biología, 15(2), 47-57. Recuperado a partir de https://revistas.unc.edu.ar/index.php/revistaadbia/article/view/22353

CÓMO CITAR ESTE ARTÍCULO

Bermúdez, G. M. A. y Occelli, M. (2020). Enfoques para la enseñanza de la Biología: una mirada para los contenidos. Didáctica de las ciencias experimentales y sociales, 39, 135-148. DOI: 10.7203/DCES.39.16854. 